\title{
Serum prevalence of celiac disease in children and adolescents with type 1 diabetes mellitus
}

\author{
Jacqueline Araújo, ${ }^{1}$ Gisélia Alves Pontes da Silva, ${ }^{2}$ Francisco Montenegro de Melo $^{3}$
}

\begin{abstract}
Objective: The association between celiac disease and diabetes mellitus has been known for many decades. This combination can be observed in a large proportion of diabetic patients, who are generally asymptomatic. The objective of this study was to evaluate the seroprevalence of celiac disease in children and adolescents with type 1 diabetes mellitus.

Methods: This was a cross-sectional study employing antibody IgA anti-transglutaminase for the serological screening of 354 diabetic children and adolescents treated at pediatric endocrinology clinics in Recife, state of Pernambuco, during the period from January to June 2004.

Results: The human anti-transglutaminase test was positive in 37/354 patients, resulting in a seroprevalence of $10.5 \%$ ( $95 \%$ CI $7.6-14.2 \%$ ). Male patients predominated (56.8\%) over female patients $(43.2 \%)$ among those that were seropositive, but without statistical significance. Anti-endomysial antibody testing was performed on patients with positive human anti-transglutaminase results, being negative in $14 / 37(37.8 \%)$ and positive in $22 / 37(59.5 \%)$.

Conclusions: The seroprevalence of celiac disease found in diabetic children and adolescents in Pernambuco is elevated, being comparable with levels observed in studies in North America and Europe and lower than in Africa, suggesting that serological screening for celiac disease should be performed for all children and adolescents with type 1 diabetes mellitus.

J Pediatr (Rio J). 2006;82(3):210-4: Type 1 diabetes mellitus, celiac disease, prevalence, children and adolescents.
\end{abstract}

\section{Introduction}

The association between celiac disease (CD) and type 1 diabetes mellitus (DM1) has been studied for almost 50 years. Many studies all over the world have demonstrated increased prevalence of $C D$ in children, adolescents and adults with DM1. ${ }^{1}$ Both are autoimmune conditions resulting from a complex interaction between genetic, immunological and environmental factors. It is not yet clear whether the simultaneous occurrence of the two diseases is linked to a common genetic base, or whether one disease in fact predisposes patients to the other; currently it is the first

1. Endocrinologista pediatra, Instituto Materno-Infantil de Pernambuco (IMIP) e Hospital das Clínicas, Recife, PE, Brasil. Mestre, Universidade Federal de Pernambuco (UFPE), Recife, PE, Brasil.

2. Professora adjunta, UFPE, Recife, PE, Brasil. Doutora, Universidade Federal de São Paulo - Escola Paulista de Medicina (UNIFESP-EPM), São Paulo, SP, Brasil.

3. Professor adjunto, Universidade de Pernambuco (UPE), Recife, PE, Brasil. Mestre, UFPE, Recife, PE, Brasil.

Manuscript received Oct 24 2005, accepted for publication Jan 182006.

Suggested citation: Araújo J, da Silva GA, de Melo FM. Serum prevalence of celiac disease in children and adolescents with type 1 diabetes mellitus. J Pediatr (Rio J). 2006;82:210-4. hypothesis that is more widely accepted, with the association of the two being explained, at least in part, by their sharing a common genetic mechanism in the human leukocyte antigen (HLA) system. ${ }^{2}$

In the past, when diagnosis was still based solely on the classic clinical manifestations, it was believed that $C D$ was rare among diabetic patients. As ever more sensitive serological markers began to be used the disease was shown to be relatively. ${ }^{3-5}$ Patients are generally asymptomatic and diagnosis is made on the basis of screening tests alone. The majority of diabetic patients exhibit the subclinical or silent form of $C D, 6$ and just $10 \%$ or so are identified through classic symptomology. ${ }^{2}$

Undiagnosed $C D$ that goes untreated is a cause for concern, since over the long term it is related with increased risks of mortality and morbidity. ${ }^{7}$ While there is no doubt about the increased prevalence of $C D$ in patients with DM1, there is still debate on whether a routine screening program should be instituted to detect subclinical or silent cases and on which serological test should be chosen if instituted. 8,9 
Several studies have been carried out to compare the sensitivity and specificity of serological tests for $C D$. Currently, it is known that the anti-endomysial antibody (AAE) and the human anti-tissue transglutaminase antibody (anti-tTG) are superior to other tests, but it is not known if there is any advantage between AAE and human antitTG. Although both tests offer elevated sensitivity and specificity, some studies have demonstrated varying degrees of agreement. ${ }^{9,10}$ Recently, the North American Society for Pediatric Gastroenterology, Hepatology and Nutrition, analyzing the literature published from 1966 to 2003, released a new consensus statement on CD and recommended human anti-tTG as the test of choice for initial screening of groups at risk of CD. ${ }^{11}$

The objective of this study was to determine the seroprevalence of $\mathrm{CD}$ in children and adolescents with prior diagnosis of DM1, using the human anti-tTG test for screening.

\section{Material and methods}

This study was carried out at the pediatric endocrinology services of the public healthcare system in Recife, during the period from January to June in 2004. The study design is cross-sectional and descriptive, analyzing children and adolescents with previous DM1 diagnosis being treated at these services.

The sample was obtained by convenience, following the demand at the clinics involved, with sample size estimate based on an observed seroprevalence of $21 \%$ in a previous study carried out in Pernambuco with a small group of diabetic children and adolescents, ${ }^{12}$ allowing for a prevalence variation of $25 \%$ either way and a confidence interval of $95 \%$. The sample size thus estimated was 231 and 361 patients were recruited. The study population was made up of diabetic patients aged 2 years to 19 years and 11 months. The age of greater than or equal to 2 years was chosen due to the probability of children in our region being exposed to gluten for at least 1 year by this age. Patients were excluded if they had IgA deficiency because of the probability of false negatives for human anti-tTG IgA.

The variables studied were: age, according to Marcondes, with children from 2 years to 9 years and 11 months and adolescents from 10 years to 19 years and 11 months included; previous diagnosis of DM1, according to American Diabetes Association criteria. Positive seroprevalence for human anti-tTG with concentrations greater than $20 \mathrm{U} / \mathrm{ml}$ and positive for $A A E$ with uniform fluorescence at a $1 / 5$ dilution of serum. ${ }^{13}$ Patients were defined as IgA deficient if they had below-normal levels for their age group. ${ }^{14}$

All diabetic patients aged 2 years to 19 years and 11 months who presented at consultations during this period were approached and, after free and informed consent had been obtained, a questionnaire was completed and a blood sample taken, by venous puncture, into tubes without anticoagulant, for centrifugal separation of serum. Samples were aliquoted and frozen at $-20{ }^{\circ} \mathrm{C}$ until the laboratory tests were carried out. Serum assays for immunoglobulin A (IgA) were performed for all patients, human anti-tTG (IgA fraction) assays were then carried out for those patients who were not IgA-deficient and then AAE (IgA fraction) assays were carried out for patients whose human anti-tTG test was positive.

The human anti-tTG IgA test was performed using the enzyme immunoassay technique (Biosystems, Spain) by microplate test, in which antibodies present in the serum bond with antigens absorbed into the surface of the polyethylene microplate wells. Care was taken to ensure that controls were set up for $10 \%$ of the samples.

The AAE test was performed using indirect immunofluorescence, using distal sections of monkey esophagus fixed on microscope slides (Biosystems, Spain). The AAE in serum bonds with the substrate and is revealed by anti-IgA antibodies marked with fluorescein isothiocyanate. ${ }^{13}$ A technician experienced in immunofluorescence performed all readings using a LEITZ microscope with a mercury lamp (Germany).

The IgA assay was performed using immunoturbidimetry, with kits provided by Biosystems (Spain), and the automated Cobas Mira Plus system used for analysis.

Patients with positive serology were referred to the pediatric gastroenterology clinic for investigation and treatment. All test results, whether positive or negative, were duly added to the medical record of each patient.

Data were stored in an Epi-Info 6.0 file. The Check module was used to automatically verify data entry errors. Data was double input and the Validate module run. Seroprevalence was calculated from the proportion of individuals with positive serology for human anti-tTG in the study population, and the confidence interval (CI) was calculated.

The project was evaluated and approved in advance by the Human Research Ethics Committee at Instituto MaternoInfantil Professor Fernando Figueira, Recife, Brazil.

\section{Results}

Three hundred and sixty-one children and adolescents with prior diagnosis of DM1 were evaluated between January 1 and 30 June, 2004. Seven individuals were excluded because they exhibited IgA deficiency, with a total of 354 patients remaining. The median age was 12 years $(\mathrm{p} 25=9$ years, $\mathrm{p} 75=15$ years), with $184 / 354$ $(52.0 \%)$ being male and $170 / 354$ (48\%) female. There 
were $172 / 354(48.6 \%)$ residents of Recife the metropolitan region and $182 / 354$ (51.4\%) from provincial Pernambuco state.

The human anti-tTG antibody test was positive in 37 of the 354 patients studied, resulting in overall seroprevalence of $10.5 \%$ ( $95 \%$ CI $7.6-14.2 \%$ ). Seroprevalence for the subset of children was $13.8 \%$ (95\% CI 7.9-21.6\%) and for the adolescents this was $9 \%$ (95\%CI 5.7-13.2\%). Children predominated over adolescents, but this difference was not statistically significant ( $p=0.2$ ).

The ages of patients with positive anti-tTG antibody tests varied from 3 years and 5 months to 19 years and 10 months (median of 11 years; p25 = 8 years, p75 $=15$ years). The male sex predominated (56.8\%) over the female sex $(43.2 \%)$, but without statistical significance $(p=0.66)$. Age at diagnosis of diabetes varied from 1 year to 12 years and 10 months, (median of 5 years; p25 = 3 years, p75 $=8$ years), and time form diabetes diagnosis to sample collection varied from 1 month to 16 years and 10 months, (median of 6 years; p25 $=2$ years, p75 $=9$ years). Around $19 \%$ (7/37) of the anti-tTG positive patients had been diagnosed with DM1 for less than 2 years at the time of collection, with $13 \%$ (5/37) having had diabetes for less than 1 year.

The AAE assay was performed for all human anti-tTG positive patients, and the results were positive in $22 / 37$ $(59.5 \%)$ and negative in $14 / 37$ patients (37.8\%) with inconclusive results in $1 / 37(2.7 \%)$. The endomysial antibody (EMA) test was positive in three patients (3/7) and negative in four (4/7) of those whose anti-tTG was positive and who had been diagnosed less than 2 years previously with DM1.

\section{Discussion}

Several studies have demonstrated a wide geographical variation in the seroprevalence of $C D$ in diabetic children and adolescents worldwide, without, however, exhibiting any link with the incidence of DM1 or CD. $3,4,6,15,16$ The highest incidence rates of DM1 in the world are in Nordic countries in Europe. ${ }^{17}$ However, studies of the seroprevalence of $C D$ in diabetic children and adolescents, using anti-gliadin antibodies (AGA) for screening, found different results in countries in the same region: in Finland around $2.4 \%{ }^{15}$ and in Denmark, 9.4\%. ${ }^{3}$ The same can be observed when European countries that used the AAE test for screening are compared, such as Hungary (11.7\%), 18 Italy $(6 \%)^{19}$ and Austria $(3.5 \%),{ }^{20}$ suggesting that the variation may be due to population characteristics that are as yet unknown. Studies of the CD seroprevalence in North America, using $A A E$ serological testing, returned more uniform results: in Canada around $8 \% 21$ and in the USA, $7 \% .{ }^{16}$ The highest seroprevalence rates in the world for CD with DM1 were observed in Africa (Algeria 13.7\% and
Libya $21.3 \%), 4,6$ although these studies used the AGA test, which is now considered a less sensitive and specific serological test.

The seroprevalence of $C D$ in diabetic children and adolescents found in the current study was lower than rates observed in countries with socioeconomic conditions similar to ours, such as in the African studies using AGA 4 or in India, with anti-tTG, ${ }^{22}$ and were similar to the USA $(11.6 \%)^{23}$ and higher than Germany $(4.4 \%),{ }^{10}$ in studies that used the human anti-tTG serological test for screening.

The cross-sectional design of this study allows the seroprevalence of $C D$ in the selected population to be determined at a given moment. It is, however, known that patients with serology that is initially negative can become positive for $C D$ over time. There is not yet consensus on the frequency with which serological tests should be repeated for diabetic patients. Some authors 24,25 recommend serological screening at the time of DM1 diagnosis and annually or biennially thereafter.

While the gold standard for the diagnosis of $C D$ remains histological findings, ${ }^{11}$ the fact that biopsy is an invasive method is an obstacle to its acceptance as a diagnostic method for initial investigation. Furthermore, the wide spectrum of $C D$ and of its non-specific clinical manifestations makes the identification of patients requiring investigation problematic. Many studies have been carried out with the objective of identifying serological screening methods with adequate sensitivity and specificity that could, in the future, substitute biopsy for the diagnosis of CD.9,10,15

Several articles have demonstrated the elevated accuracy of AAE. 9,13 However, there are certain limitations to its use on a large scale, such as the elevated cost, laborious technique and the fact that it is a semi-quantitative method, subject in part to observer interpretation. Furthermore, it has recently been observed that there may be a correlation between the degree of villous atrophy and positive AAE tests, which may be negative in patients with only partial alterations to the intestinal mucosa. ${ }^{26}$ As a result of these problems, the human anti-tTG test has been suggested as the test of choice for initial screening. In addition to comparable sensitivity and specificity to $A A E$, the method is much simpler, and a large number of samples can be analyzed and with lower costs. ${ }^{9}$ Some authors have observed that, in patients with DM1 with and the silent form of $C D$, the anti-tTG test is more sensitive than AAE. ${ }^{10}$ On the other hand, the elevated number of false-positives in some studies using human anti-tTG has led some authors to suggest a new protocol using human anti-tTG for initial screening and AAE for positive cases. ${ }^{27}$ In a recent publication, the a North American Society for Pediatric Gastroenterology, Hepatology and Nutrition recommends human anti-tTG as the test of choice for initial screening in high-risk groups for $C D$, followed by 
intestinal biopsy. Only when histological findings are not consistent with $C D$ should $A A E$, HLA assay or a repeat biopsy be considered. ${ }^{11}$

For the present study, the decision was taken to use sequential serology, with human anti-tTG for initial screening, followed by AAE testing of positive patients. We observed that $37 \%$ were anti-tTG positive, but AAE negative. Furthermore, we found positive EMA results in around $43 \%$ of the anti-tTG positive patients who had had diabetes for less than 2 years at the time samples were collected. The presence of EMA in patients with little time of DM1 diagnosis could suggest a higher probability of CD, since this antibody appears to be related with the degree of mucosal injury. It is only through follow-up of these patients and confirmation of histological findings that it will be possible to reach conclusions whether these results represent an elevated rate of false-positive results from anti-tTG testing, or, in contrast, the increased sensitivity of the method.

There is strong evidence for the association between IgA deficiency and $C D$, with a frequency of approximately $2 \%$, which is 10 to 15 times more common than in the general population. ${ }^{28}$ Individuals with IgA deficiency and $C D$ do not form anti-IgA antibodies, and the serological tests for human anti-tTG and $A A E$ commercially available are habitually limited to the IgA isotopes in antibodies. Serum IgA assay was included in the battery of tests in this study and it was observed that deficiency was confirmed in $7 / 361(1.9 \%)$, with these patients being excluded from the study because of the chance of false-negative serology for CD.

There are suggestions that $C D$ may be more prevalent among older age groups, due to greater length of gluten exposure. ${ }^{6}$ In high-risk groups such as DM1 patients, this association has not been confirmed, since other factors may be involved, such as age at diagnosis of diabetes and the time with diabetes at the time of testing. In this study, there was a predominance of seroprevalence in the subset of children when compared with the adolescents, without statistical significance $(p=0.2)$. One possible explanation is that the estimated sample size, for children and adolescents, was not sufficient for an analysis by age group. Another possibility is that age at diagnosis of diabetes is an important factor in the prevalence of CD. 20 Studies of children who had been diagnosed with DM1 before 5 years of age have demonstrated more accentuated autoimmunity, with more frequent presence of pancreatic islet cell antibodies and insulin autoantibodies than in the higher age group, in addition to accentuated genetic susceptibility demonstrated by more frequent associations with the genotype at risk of the HLA system defect, ${ }^{29}$ leading to the belief that children who are younger at diagnosis may present a more elevated risk of developing other similar autoimmune diseases and biologically-based diseases like CD.

Calliari ${ }^{30}$ states that the association between $C D$ and DM1 should receive greater attention in the care of diabetic patients and proposes the performance of multicenter studies in Brazil. The seroprevalence of 10.5\% observed in the current study is elevated, suggesting that screening for $C D$ should be routinely undertaken for children and adolescents with DM1. Nevertheless, it is only after intestinal biopsy of these patients and the calculation of $C D$ prevalence based on histological findings will it be possible to establish the value of human anti-tTG for screening our population.

\section{Acknowledgements}

This research was made possible by funds for purchasing the serological kits from the NOVO NORDISK laboratory.

We are grateful to the team at the Diva Montenegro Pathology and Clinical Analysis Laboratory, Prof. Francisco Montenegro and Suely Lyra, for their dedication to this project.

We would also like to thank Dr. Elcy Falcão, Dr. Amélia Melo and Dr. Cíntia Waechter, for helping with enrolling patients.

\section{References}

1. Koletzko S, Burgin-Wolff A, Koletzko B, Knapp M, Burger W, Gruneklee D. Prevalence of coeliac disease in diabetic children and adolescents. A multicentre study. Eur J Pediatr. 1988;148:113-7.

2. Collin P, Kaukinen K, Valimaki M, Salmi J. Endocrinological disorders and celiac disease. Endocr Rev. 2002;23:464-83.

3. Hansen D, Bennedbaek FN, Hansen LK, Hoier-Madsen M, Hegedu $\mathrm{LS}$, Jacobsen $\mathrm{BB}$, et al. High prevalence of coeliac disease in Danish children with type 1 diabetes mellitus. Acta Paediatr. 2001; $90: 1238-43$.

4. Ashabani A, Abushofa U, Abusrewill S, Abdelazez M, Tuckova L, Tlaskalova-Hogenova $H$. The prevalence of coeliac disease in Libyan children with type 1 diabetes mellitus. Diabetes Metab Res Rev. 2003;19:69-75

5. Boudraa G, Hachelaf W, Benbouabdellah M, Belkadi M, Benmansour FZ, Touhami M. Prevalence of coeliac disease in diabetic children and their first- degree relatives in west Algeria: screening with serological markers. Acta Paediatr Suppl. 1996;412:58-60.

6. Brook LS. Diagnosing celiac disease in 2002: who, why, and how? Pediatrics. 2002;109:952-4.

7. West J, Logan RF, Smith CJ, Hubbard RB, Card TR. Malignancy and mortality in people with coeliac disease: population based cohort study. BMJ. 2004;329:716-9.

8. Schober E, Rami B, Granditsch G, Crone J. Coeliac disease in children and adolescents with type 1 diabetes mellitus: to screen or not, to treat or not? Horm Res. 2002;57 Suppl $1: 97-100$.

9. Carroccio A, Vitale G, Di Prima L, Chifari N, Napoli S, La Russa $C$, et al. Comparison of anti-transglutaminase ELISAs and an anti-endomysial antibody assay in the diagnosis of celiac disease: a prospective study. Clin Chem. 2002;48:1546-50.

10. Kordonouri O, Dieterich W, Schuppan D, Webert G, Muller C, Sarioglu N, et al. Autoantibodies to tissue transglutaminase are sensitive serological parameters for detecting silent coeliac disease in patients with type 1 diabetes mellitus. Diabet Med. 2000;17:441-4. 
11. Hill ID, Dirks MH, Liptak GS, Colleti RB, Fasano A, Guandalini S et al. Guidelines for the diagnosis and treatment of celiac disease in children: recommendations of the North American Society for Pediatric Gastroenterology, Hepatology and Nutrition. J Pediatr Gastroenterol Nutr. 2005;40:1-19.

12. Brandt KG, Silva GAP, Antunes MC. Doença celíaca em um grupo de crianças e adolescentes portadores de diabetes mellitus tipo 1. Arq Bras Endocrinol Metabol. 2004;48:823-7.

13. Grodzinsky E, Hed J, Skoght T. IgA antiendomysium antibodies have a high positive predictive value for celiac disease in asymptomatic patients. Allergy. 1994;49:593-7.

14. Naspitz CK, Solé D, Sampaio C, Magda MS, Gonzalez CH. Níveis séricos de IgG, IgM, IgA em crianças brasileiras normais. J Pediatr (Rio J). 1982;52:121-6.

15. Saukkonen $T$, Savilahti E, Reijonen H, Honen J, Tuomilehto-Wolf E, Akerblom HK. Coeliac disease: frequent occurrence after clinical onset of insulin dependent diabetes mellitus. Childhood Diabetes in Finland Study Group. Diabet Med. 1996;13:464-70.

16. Aktay AN, Lee PC, Kumar V, Parton E, Wyatt DT, Werlin SL. The prevalence and clinical characteristics of celiac disease in juvenile diabetes in Wisconsin. J Pediatr Gastroenterol Nutr. 2001;33:462-5.

17. Karvonen M, Viik-Kajander M, Moltchanova E, Libman I, LaPorte $\mathrm{R}$, Tuomilehto J. Incidence of childhood type 1 diabetes worldwide. Diabetes Mondiale (DiaMond) Project Group. Diabetes Care. 2000;23:1516-26.

18. Arato A, Korner A, Veres G, Dezsofi A, Ujpal I, Madacsy L. Frequency of coeliac disease in Hungarian children with type 1 diabetes mellitus. Eur J Pediatr. 2003;162:1-5.

19. Barera G, Bianchi C, Calisti L, Cerutti F, Dammacco F, Frezza E, et al. Screening of diabetic children for coeliac disease with antigliadin antibodies and HLA typing. Arch Dis Child. $1991 ; 66: 491-4$.

20. Schober EB, Bittmann G, Granditsch WD, Huber A, Huppe A, Jager $G$, et al. Screening by anti-endomysium antibody for celiac disease in diabetic children and adolescents in Austria. J Pediatr Gastroenterol Nutr. 2000;30:391-6.

21. Fraser-Reynolds KA, Butzner JD, Stephure DK, Trussell RA, Scott RB. Use of immunoglobulin A-antiendomysial antibody to screen for celiac disease in North American children with type 1 diabetes. Diabetes Care. 1998;21:1985-9.

22. Kanungo A, Shtauvere-Brameus A, Samal KC, Sanjeevi CB. Autoantibodies to tissue transglutaminase in patients from eastern India with malnutrition-modulated diabetes mellitus, insulin-dependent diabetes mellitus, and non-insulin-dependent diabetes mellitus. Ann N Y Acad Sci. 2002;958:232-4
23. Bao F, Yu L, Babu S, Wang T, Hoffenberg EJ, Rewers M, et al. One third of HLA DQ2 homozygous patients with type 1 diabetes express celiac disease-associated transglutaminase autoantibodies. J Autoimmun. 1999;13:143-8.

24. Barera G, Bonfanti R, Viscardi M, Bazzigaluppi E, Calori G, Meschi $F$, et al. Occurrence of celiac disease after onset of type 1 diabetes: a 6-year prospective longitudinal study. Pediatrics. 2002;109:833-8.

25. Glastras SJ, Craig ME, Verge CF, Chan AK, Cusumano JM, Donaghue KC. The role of autoimmunity at diagnosis of type 1 diabetes in the development of thyroid celiac disease and microvascular complications. Diabetes Care. 2005;28:2170-5.

26. Abrams JA, Diamond B, Rotterdam H, Green PH. Seronegative celiac disease: increased prevalence with lesser degrees of villous atrophy. Dig Dis Sci. 2004;49:546-50.

27. Lock RJ, Stevens S, Pitcher MC, Unsworth DJ. Is immunoglobulin A anti-tissue transglutaminase antibody a reliable serological marker of coeliac disease? Eur J Gastroenterol Hepatol. 2004; 16:467-70

28. Cataldo F, Marino V, Ventura A, Bottaro G, Corazza GR. Prevalence and clinical features of selective immunoglobulin A deficiency in coeliac disease: an Italian multicentre study. Italian Society of Paediatric Gastroenterology and Hepatology (SIGEP).Gut. 1998;42:362-5.

29. Komulainen J, Kulmala P, Savola K, Lounamaa R, Ilonen J, Reijonen $H$, et al. Clinical, autoimmune, and genetic characteristics of very young children with type 1 diabetes. Childhood Diabetes in Finland. Diabetes Care. 1999;22:1950-5.

30. Calliari LE. A auto-imunidade extra-pancreática no DM1: pesquisando também a doença celíaca. Arq Bras Endocrinol Metabol. 2004;48:785-6.

Correspondence:

Jacqueline Araújo

Rua Dhália, 304/902, Boa Viagem

CEP 51020-290 - Recife, PE - Brazi

Tel.: +55 (81) 3326.0891, +55 (81) 3445.1145

E-mail: araujoj@hotlink.com.br 